\title{
La gestión del conocimiento es ya una realidad para la empresa española
}

\author{
Por Joaquín García-Tapial Arregui
}

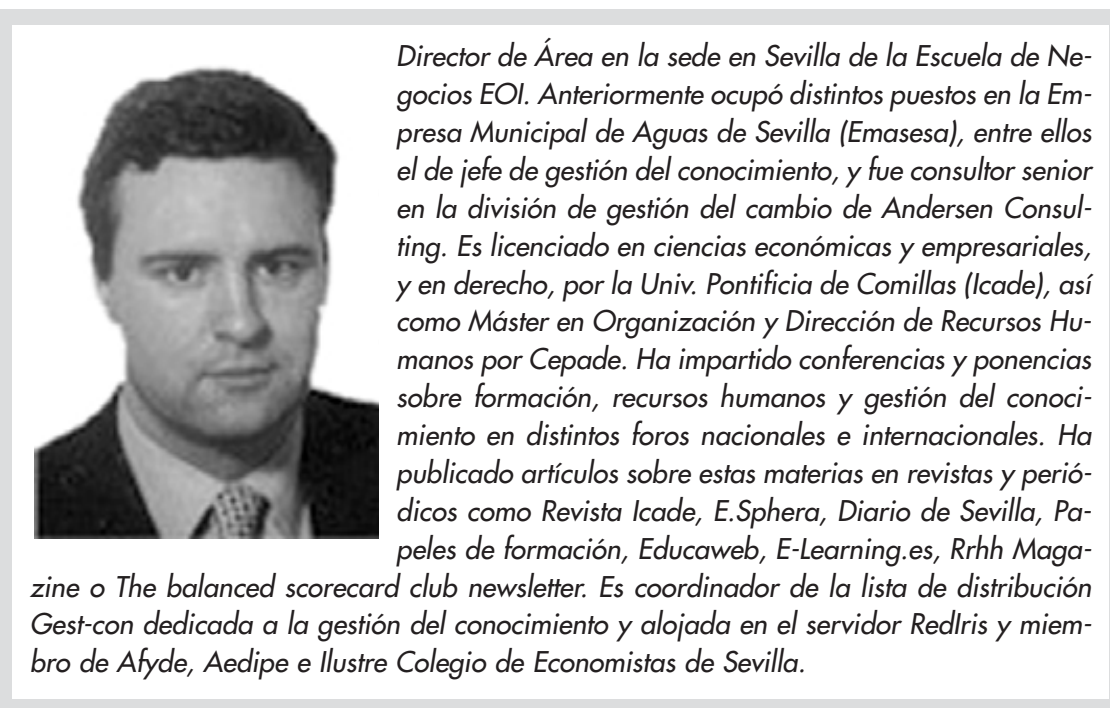

Resumen: El presente artículo tiene como finalidad presentar el estado actual de la gestión del conocimiento en la empresa española, su grado de implantación y los planes de futuro, haciendo especial hincapié en la pequeña y mediana empresa. En este sentido, se proponen varias iniciativas para que las mismas "rentabilicen" su conocimiento. El artículo se basa en el estudio realizado por EOI (Escuela de Organización Industrial) en el año 2001 sobre una muestra de más de 300 empresas.

Palabras clave: Gestión del conocimiento, Capital intelectual, E-business, EOI, Pyme, Innovación. 


\section{En primera línea en Sistemas de Información y Gestión del Conocimiento.}

[ Informática Documental

- Internet, Intranet

Ediciôn de Bases de Datos en CD-Rom

- Sistema Integrado de Gestión Bibliotecaria Absys

Catalogación Retrospectiva

- Sistema Integrado de Gestión de Centros Archivisticos Albalá

Raimundo Fernández Villaverde, 28

28003 Madrid (España)

Teléfono +34914560360 - Fax +34915330958

www.baratz.es - E-mail: informa@baratz.es

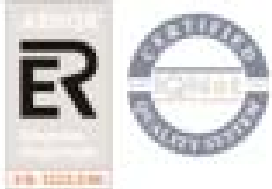




\section{Title: Knowledge management is now a reality in Spanish companies}

Abstract: The present article discusses the manner and extent of the implementation of knowledge management in Spanish companies. The focus is on small enterprises, with proposed initiatives regarding the way for SMEs to take advantage of their own accumulated knowledge. The article is based on research performed on more than 300 companies during 2001 by the EOI (School of Industrial Organisation) Business School.

Keywords: Knowledge management, Intellectual capital, E-business, EOI, Small business, SME, Innovation.

HABITUALMENTE VENIMOS LEYENDO y escuchando que, en el entorno actual, las personas se convierten en el principal activo de una organización. Su valor ya no reside en sus bienes tangibles, sino en los conocimientos técnicos y especializados de su personal, en su experiencia, en la propiedad intelectual, la fidelidad de los clientes, etc. En resumen, en lo que se ha venido en llamar capital intelectual o conocimiento.

Ante estas nuevas "reglas del juego", la empresa que aproveche los efectivos intelectuales de la organización, que desarrolle su capacidad de aprendizaje, que potencie la innovación constante y la creación de nuevos conocimientos, estará en situación de afrontar los retos futuros. Capturar y gestionar estas capacidades, estos conocimientos, y rentabilizarlos es lo que denominamos gestión del conocimiento (GC).

\section{Algunas cifras}

Todo este proceso, así definido, está adquiriendo cada vez más importancia para las empresas. De hecho, el 47,3\% de las compañías españolas entienden que en estos momentos su ventaja competitiva se basa en primer lugar en el saber de sus trabajadores, frente a otros factores como la tecnología o la capacidad financiera. $\mathrm{Y}$ si se les pide que mencionen 3 principios de competitividad, el conocimiento aparece en el $81 \%$ de las respuestas.

También es relevante comprobar cómo el $78 \%$ de estas organizaciones que consideran el conoci- miento como la principal fuente de desarrollo documentan sus procedimientos internos, pero sólo en un $32 \%$ cuentan con sistemas que gestionen dicho conocimiento (aunque este porcentaje aumenta hasta el $69 \%$ si se les pregunta si tienen previsto implantarlo en los próximos 3 años).

Sin embargo, la situación cambia cuando se trata de llevar estas opiniones a la práctica. Así, según la encuesta realizada por $E O I$ en el año 2001, solamente el $34 \%$ de las empresas españolas disponen en la actualidad de una persona o unidad dedicada a la GC, aunque este porcentaje aumenta hasta el $47 \%$ cuando se trata de compañías con más de 100 empleados, y al $42 \%$ cuando la facturación es superior a los 12 millones de euros. Así, a mayor número de empleados/mayor facturación aumenta el interés por la cuestión. En cuanto a los sectores de actividad es el financiero el que presenta una mayor preocupación por la materia y el agroalimentario el que menos.

En lo que se refiere a su perfil, aquellas empresas que disponen de unidades de GC, sólo en un 57,8\% disponen de departamento de rrhh (lo cual es sorprendente y nos indica cómo existe una tendencia a que sean los departamentos de GC los que asuman la gestión de los recursos humanos dentro de la empresa), que en un $84,3 \%$ tienen documentados sus procedimientos internos y que en el $73,5 \%$ de los casos realizan transacciones electrónicas vía internet (lo que quiere decir que poseen vocación por el ebusiness).

\section{«La inversión en recursos huma- nos, técnicos y económicos que requiere un sis- tema de GC es, a priori, grande»}

Si profundizamos un poco más intentando conocer si se dispone de un sistema de GC, vemos cómo los resultados son coherentes con los anteriores. Así, sólo el 27,7\% de las empresas los tienen instalados (lo que supone el $80 \%$ de las que contaban con un responsable de GC), siendo las del sector financiero y de telecomunicaciones las que presentan un mayor porcentaje de implantaciones. Además, son

\begin{tabular}{|l|l|l|}
\hline & \multicolumn{1}{|c|}{ SÍ } & \multicolumn{1}{c|}{ NO } \\
\hline ¿Disponen de departamento de recursos humanos? & $48,6 \%$ & $51,4 \%$ \\
\hline ¿Tienen documentados los procedimientos internos? & $78,2 \%$ & $21,8 \%$ \\
\hline ¿Consideran el comercio electrónico como vía de crecimiento? & $50,4 \%$ & $49,6 \%$ \\
\hline ¿Realizan transacciones vía internet? & $74,6 \%$ & $25,4 \%$ \\
\hline ¿Tienen implantado un sistema de gestión del conocimiento? & $32,9 \%$ & $67,1 \%$ \\
\hline ¿Tienen previsto implantarlo en los próximos 3 años? & $42,6 \%$ & $30 \%$ \\
\hline
\end{tabular}

Características de las empresas que consideran el conocimiento de sus trabajadores como su principal ventaja competitiva

Fuente: EOI, Encuesta sobre gestión del conocimiento, 2001 
las compañías de mayor dimensión también las que más utilizan estos sistemas.

Significativo es que, del $64,7 \%$ que no utilizan ningún sistema, el $30 \%$ manifiesta su intención de implantarlo en los próximos 3 años, con lo que cerca del $50 \%$ de las empresas dispondrían de alguna herramienta de esta índole. Además, el 56\% de los que lo tienen ya en uso están dispuestos a renovar/actualizarlo en este plazo.

Otro dato interesante es que el $86,7 \%$ de las empresas que disponen de un sistema de GC, y el $88,4 \%$ de las que tienen previsto implantar uno, ya tienen documentados sus procedimientos internos, con lo que parece evidente que éste es un primer paso cara a su instalación. Estos porcentajes aumentan al $95,2 \%$ y $90,2 \%$ cuando se trata de empresas que tienen almacenada la información de sus clientes en bases de datos. El 100\% de las compañías que tienen implantado un sistema de GC y el $94 \%$ de las que tienen previsto hacerlo en los próximos 3 años tienen detectadas sus competencias clave, requisito imprescindible para realizar estas acciones.

Finalmente es relevante que el $32 \%$ de las empresas que mencionan el conocimiento como ventaja competitiva tengan implantado un sistema para gestionarlo, porcentaje superior al de los que consideran otras ventajas competitivas. También es importante que el $44 \%$ de los que consideran la capacidad de innovación como su ventaja competitiva tengan estos sistemas implantados.

\section{Métodos y técnicas}

Cuando las empresas hablan de herramientas y sistemas de GC están haciendo mención a un rango amplio de soluciones, que podrían agruparse en 4 apartados:

\begin{tabular}{|l|l|l|l|}
\hline & \multicolumn{1}{|c|}{ SÍ } & \multicolumn{1}{c|}{ NO } & NS/NC \\
\hline Total nacional & $27,7 \%$ & $68,7 \%$ & $3,7 \%$ \\
\hline Por sectores & & & \\
\hline Consultoría & $32,4 \%$ & $64,7 \%$ & $2,9 \%$ \\
\hline Servicios públicos & $32,6 \%$ & $65,2 \%$ & $2,2 \%$ \\
\hline Turismo & $32 \%$ & $68 \%$ & - \\
\hline Industria agroalimentaria & $17 \%$ & $78,7 \%$ & $4,3 \%$ \\
\hline Industria química & $27,7 \%$ & $66,2 \%$ & $6,2 \%$ \\
\hline Financiero & $38,1 \%$ & $57,1 \%$ & $4,8 \%$ \\
\hline Telecomunicaciones & $46,7 \%$ & $53,3 \%$ & - \\
\hline Por número de empleados & & & \\
\hline $10-25$ & $18,6 \%$ & 76,35 & $5,1 \%$ \\
\hline $26-50$ & $21,7 \%$ & $76,5 \%$ & $1,7 \%$ \\
\hline $51-100$ & $33,3 \%$ & $60,9 \%$ & $5,8 \%$ \\
\hline+100 & $42,1 \%$ & $54,4 \%$ & $3,5 \%$ \\
\hline Por facturación & & & \\
\hline $1,5-3$ millones euros & $17,3 \%$ & $77,3 \%$ & - \\
\hline $3-6$ millones euros & $27,1 \%$ & $72,9 \%$ & - \\
\hline $6-12$ millones euros & $26,3 \%$ & $71,1 \%$ & $2,6 \%$ \\
\hline $12-18$ millones euros & $36,4 \%$ & $60,6 \%$ & $3 \%$ \\
\hline
\end{tabular}

Empresas que han implantado un sistema de gestión del conocimiento (porcentaje) Fuente: EOl, Encuesta sobre gestión del conocimiento, 2001

-Comunidades de práctica: su finalidad es proporcionar foros donde se comparta conocimiento en una materia (lo que se denomina la "comunidad"). Así, en aquellas empresas donde la organización sea matricial en lugar de funcional, habrá personas con conocimientos similares o relacionados en distintas áreas de la misma, lo que dificulta que se comparta la información y que unos sectores se aprovechen de la experiencia y conocimientos de otro.

- Sistemas de toma de decisiones: tienen como finalidad ayudar a las personas que se encuentran en la organización a un nivel ejecutivo, proporcionándoles información acerca de experiencias pasadas similares (en la propia empresa o fuera de ella) que le puedan aportar elementos de juicio suficientes como para asegurar que la decisión tomada es la idónea.

-Desarrollo de competencias: bajo este epígrafe se engloban las herramientas que permiten la creación de conocimiento individual. No debe entenderse que las mis- mas son el equivalente a la formación tradicional, sino que van más allá, puesto que permiten la puesta a disposición de toda la organización del conocimiento de los expertos en cada una de las materias (o "competencias"). Un ejemplo de lo que estamos hablando sería el aprendizaje basado en el estudio del caso.

-Arquitectura del conocimiento: por último, existe una serie de herramientas que articulan la comunicación y la información (y, por tanto, cómo se almacena, comparte y distribuye el conocimiento) dentro de la organización. Todo ello es lo que se denomina arquitectura del conocimiento y abarca elementos tales como sistemas de gestión de bases de datos, intranets, etc.

\section{Dónde se implanta}

Debemos resaltar la diversidad de sectores. Así, encontramos desde grandes empresas de servicios (utilities) hasta hospitales, pasando por ONG's, empresas públicas, organismos del Estado, entidades fi- 


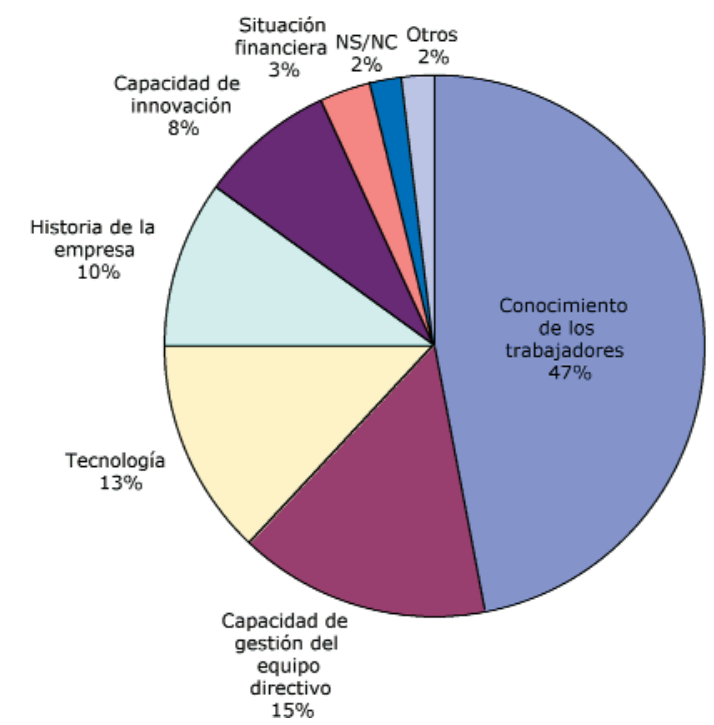

Factores en los que las empresas basan su ventaja competitiva en primer lugar

Fuente: EOI, Encuesta sobre gestión del conocimiento, 2001

nancieras, consultoras, empresas de servicios, etc., lo que nos da una idea de las posibilidades que ofrecen. Además, vemos cómo se adoptan distintos enfoques (desde la gestión documental "avanzada" hasta la creación de nuevas unidades de negocio), todos ellos válidos. Tan sólo se trata de aunar las posibilidades que ofrecen estas herramientas con las necesidades de negocio de cada organización.

Todo lo expuesto hasta ahora se encuentra con un gran inconveniente. La inversión en recursos humanos, técnicos y económicos que requiere un sistema de GC es, a priori, grande. Por tanto, parece que se trata de una filosofía reser- vada a las grandes empresas. ¿Qué ocurre entonces con la pyme, que supone más del $90 \%$ del entramado empresarial del país?

\section{Oportunidades para las pymes}

Desde nuestro punto de vista son tantas que la pequeña y mediana empresa también debería beneficiarse de diversas maneras, tanto a nivel individual como colectivamente. Individualmente, la pyme necesita concienciarse de que la GC debe impregnar su gestión. Si consideramos el ciclo del conocimiento, cualquier pyme por pequeña que sea deberá preocuparse por:
-Identificar conocimiento: independientemente del tamaño de la empresa, es fundamental que ésta identifique sus conocimientos clave sobre los que se va a apoyar a la hora de establecer una ventaja competitiva para el futuro. Ellos serán los que determinen las actividades a desarrollar, mantener o eliminar, las inversiones a realizar, estrategias, etc.

- Crear conocimiento: el segundo aspecto con el que una pyme puede sacar provecho está en la creación de conocimiento. Precisamente el enfoque que desde ésta se da a esta actividad, yendo más allá de la formación tradicional, es el que permite que el pequeño empre-

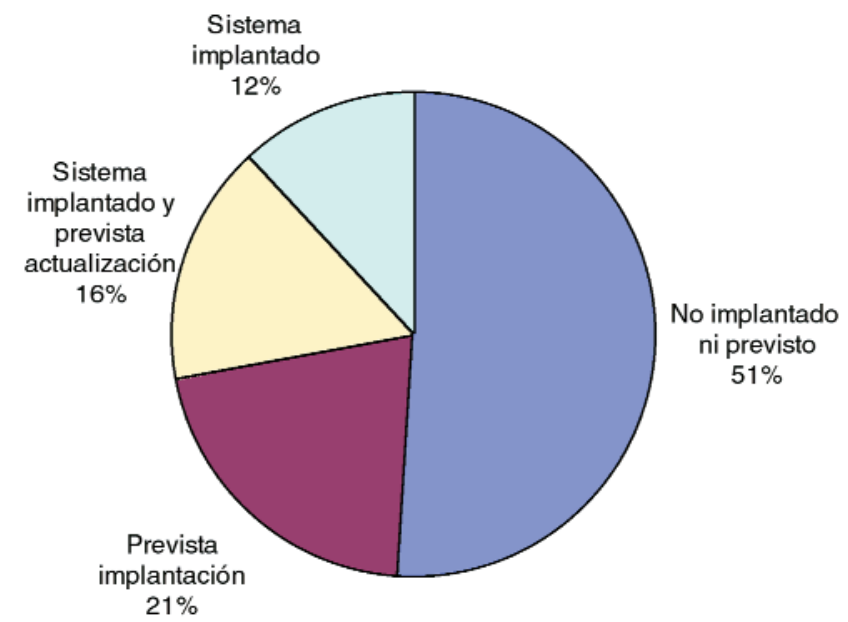

Implantación de sistemas de gestión del conocimiento en empresas españolas (porcentaje)

Fuente: EOI, Encuesta sobre gestión del conocimiento, 2001

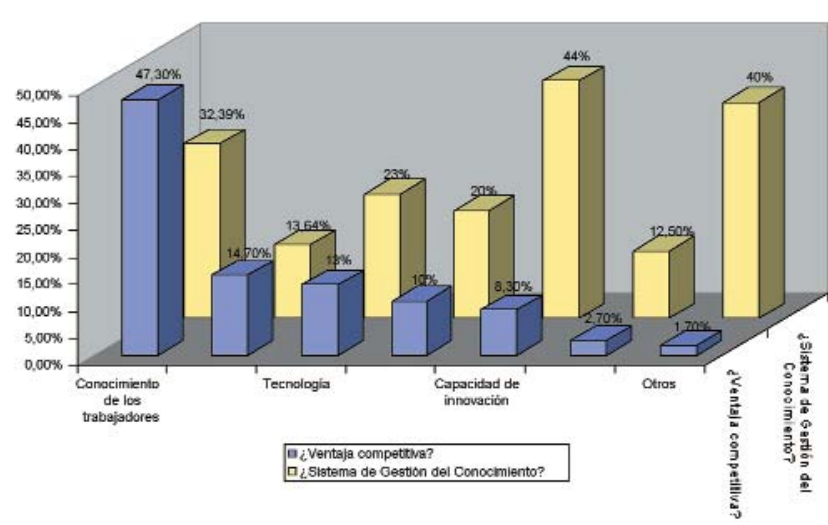

Ventajas competitivas y sistemas de gestión del conocimiento (relación)

Fuente: EOI, Encuesta sobre gestión del conocimiento, 2001 


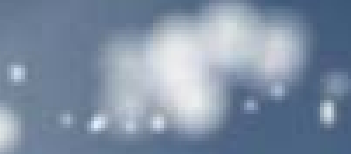

$+$

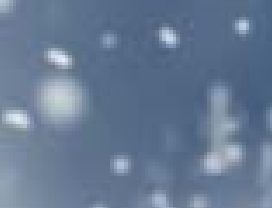

$+8$

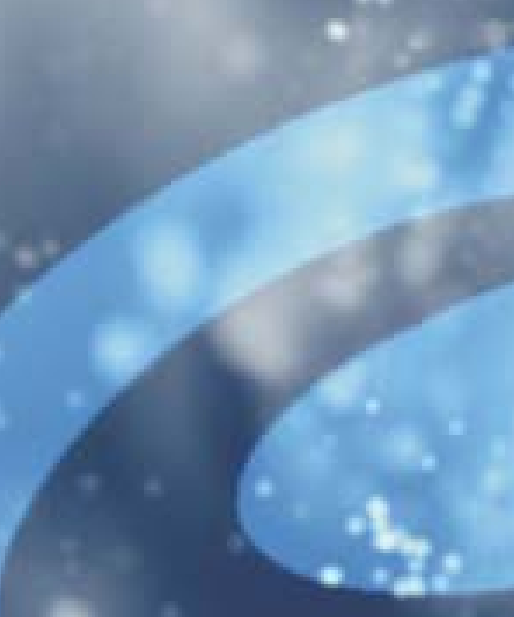

Un universo de información

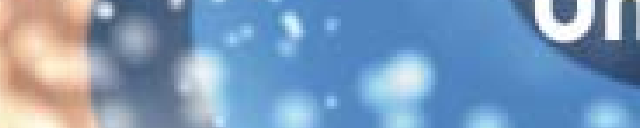

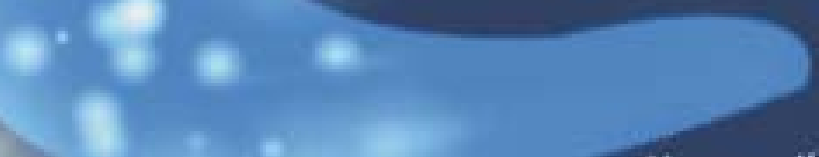

Hoy en dia, Internet es el presente y futuro en el contexto general de los sistemas de información y gestión del conocimiento. Ahora es el momento de consolidar el potencial de su biblioteca.

SIRSI es pionera en sistemas de gestión de información

y tecnologia para bibliotecas, archivos y centros de documentación. En SIRSI desarrollamos y facilitamos a nuestros clientes las herramientas más innovadoras y todos los servicios necesarios para que éstos puedan aportar a sus usuarios información y conocimiento desde cualquier fuente, en cualquier momento y lugar.

Unicorn, el sistema integrado de gestión bibliotecaria de SIRSI, aporta a los bibliotecarios una infraestructura de gestión global para controlar todos los aspectos diferenciadores de su biblioteca y facilitar e incrementar la eficiencia en el servicio al usuario final.

iBistro es la biblioteca electrónica de SIRSI que une las caracteristicas mas innovadoras a las clásicas funcionalidades del catálogo público - OPAC: reseñas, información bibliotecaria, técnicas avanzadas de búsqueda y personalización, integración de fuentes de información internas y externas a la biblioteca...

\section{Ponemos el universo del conocimiento en sus manos}

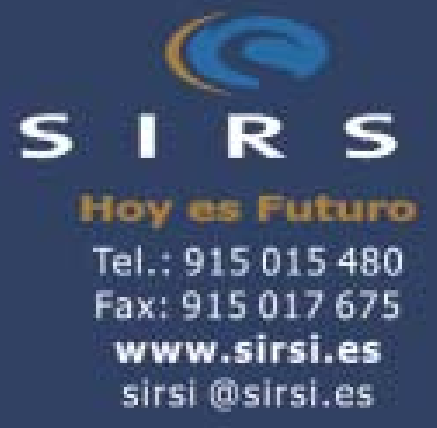


sariado se beneficie del mismo. Se trata ya no sólo de estar permanentemente al día y no descuidar la formación continua tanto propia como de su personal, sino de utilizar las fuentes de conocimiento que tiene a su disposición a un coste mínimo y que puede incorporar a la gestión diaria. Así, gracias a internet, el empresario puede acceder a un volumen de información y conocimiento inimaginable hasta hace muy poco.

-Almacenar y estructurar conocimiento: quizás sea en este punto donde el pequeño empresario tenga mayores dificultades en actuar individualmente y la necesidad de cooperación sea más grande. No obstante, sí existe una vertiente de la GC que permite su actuación de manera individual. Se trata de todo lo referido al capital relacional y, más en concreto, con proveedores y clientes. La popularización de las tecnologías de la información, permite que el pequeño empresario pueda almacenar y estructurar el conocimiento que tiene sobre sus clientes y proveedores, utilizando sencillas herramientas informáticas (basta una simple hoja de cálculo o una pequeña base de datos en Access). Este conocimiento, estructurado y explotado adecuadamente, puede suponerle una reducción de sus costes de aprovisionamiento por un lado $\mathrm{y}$ un incremento de la eficiencia de su acción comercial (por ejemplo, centrándola en aquellos clientes o sectores más rentables).

Por otro lado, como ya hemos afirmado, la GC puede tener una mayor utilidad y aplicación práctica para la pyme si se plantea de manera colectiva. ¿A qué nos estamos refiriendo? A que un colectivo de pymes, con intereses comunes y asociadas entre sí pueden compartir conocimiento entre ellas, de la misma manera que lo hacen las grandes empresas, y asumir los costes de implantar estas herra- mientas que individualmente no pueden. De esta forma, y de manera colectiva, podrían colaborar mutuamente en la creación, almacenamiento, estructuración y distribución de conocimiento entre ellas. A modo de ejemplo, ofrecemos algunos casos de pymes en los que podría darse esta agrupación:

-Que pertenezcan a un mismo sector de actividad pero que, por su implantación geográfica, no compitan entre sí.

\section{«La GC puede tener una mayor utilidad y aplica- ción práctica pa- ra la pyme si se plantea de ma- nera colectiva»}

-Que desarrollen su actividad en un mismo marco geográfico, pero que, por realizar tareas diferentes, no compitan entre sí.

-Que pertenezcan a un mismo sector de actividad y que desarrollen su actividad en el mismo marco geográfico. En este caso, pese a ser competencia las unas de las otras sí que existirían posibles áreas de interés común en las que, como sector, podrían colaborar y beneficiarse mutuamente especialmente frente a terceros. Se trataría de extrapolar experiencias como las de los consorcios de exportación al ámbito de la GC.

\section{Barreras a la implantación de la GC}

Son de naturaleza muy diversa.

\section{Económicas:}

-Alto coste de los proyectos debido a que conllevan la creación de unidades o departamentos, contratación de consultores externos, compra de herramientas tecnológicas, etc.
-Por lo habitual, es una filosofía que requiere tiempo para su implantación.

- Junto con lo anterior, en muchas empresas se buscan beneficios inmediatos y en este caso se trata de inversiones en las que el retorno generalmente se produce a medio y largo plazo.

-A menudo se precisa una "reingeniería" de procesos internos, con el coste que supone toda reorganización de la empresa.

2. Tecnológicas:

-Realización de sistemas complejos, lo que dificulta enormemente su utilización.

-Muchas empresas todavía ven la GC como una solución meramente tecnológica, lo que lleva a que se diseñen sistemas de poca aplicación "real".

-Ausencia de la infraestructura tecnológica necesaria para su implantación.

- Sistemas de información dispersos en distintas plataformas tecnológicas (necesidad de integrarlos).

-Exceso de niveles de acceso a la información.

\section{Humanas:}

Son las más difíciles de salvar.

- No se han descubierto todavía todas las posibilidades de la GC por parte de la alta dirección, lo que hace que su apoyo sea tibio o inexistente y no haya una estrategia bien definida.

—Falta de tiempo por parte de las personas para utilizarla.

-Insuficiente comunicación interna del sistema de GC implantado, lo que contribuye a su baja utilización.

- Miedo de las personas a compartir aquello que saben. En ocasiones esto se ve reforzado por la propia cultura organizativa, que 
fomenta los "silos" de información por el bajo compromiso de los empleados con la organización.

-Y, sobre todo, el cambio de mentalidad que requiere la utilización de estos sistemas.

\section{Motivación}

El papel de la Administración y de las asociaciones de empresarios en operaciones de este tipo es clave, puesto que pueden ejercer un liderazgo y actuar de catalizadores de iniciativas. Además, pueden contribuir a difundir entre el pequeño empresariado la importancia de la GC y motivarles para su participación. Incluso en una primera fase, deben ayudar a dinamizar los sistemas implantados, incentivando su utilización por parte de las empresas. Finalmente, pueden financiar proyectos. Un ejemplo de cómo puede llegar a articular una colaboración en este senti- do lo constituye el Cluster del Conocimiento en Gestión Empresarial existente en el País Vasco.

\section{Conclusiones}

La empresa española se encuentra ante una oportunidad de negocio enmarcada dentro de las denominadas nuevas tecnologías de la información. Pero no sólo es eso, sino mucho más. Se trata de un nuevo modelo de negocio, y el objetivo de las organizaciones que apuesten por él no debe ser sólo crear una página web, una intranet o un portal, mediante el cual se capitalice y se distribuya el conocimiento internamente o a terceros, sino crear una nueva organización que gire en torno al conocimiento de sus componentes, que convierta dicho capital intelectual en estructural y que genere capital financiero en base al mismo. En definitiva, una empresa que transforme el conocimiento en su principal ventaja competitiva. Y si, como país, somos capaces de hacer esto, seguramente aumentaremos nuestra eficiencia y contribuiremos al incremento de la competitividad de nuestro tejido empresarial, con todo lo que ello conlleva (empleo, riqueza, etc.).

Como se ve, apenas hemos entrado en la era de la información y rápidamente se nos demanda que pasemos a la era del conocimiento, en la que las organizaciones se van a basar en lo que saben hacer, no en lo que producen. Esto demanda un cambio urgente y de gran escala. Como toda transformación cultural, va a suponer un gran salto en el que los riesgos van a ser importantes y muchos caerán por el camino.

Joaquín García-Tapial Arregui, Director de Área, EOI Andalucía.

jgtapial@eoi.es 